Miriam Potrony, Celia Badenas, Bénédicte Naerhuyzen, Paula Aguilera, Joan Anton Puig-Butille, Gemma Tell-Marti, Alba Díaz, Cristina Carrera, Llucia Alos, Nicolas Delahaye, Josep Malvehy and Susana Puig*

\title{
Time and tumor type (primary or metastatic) do not influence the detection of BRAF/NRAS mutations in formalin fixed paraffin embedded samples from melanomas
}

DOI 10.1515/cclm-2015-1048

Received October 28, 2015; accepted March 10, 2016; previously published online April 21, 2016

\section{Abstract}

Background: $B R A F$ and NRAS mutation detection is crucial for advanced melanoma treatment. Our aim was to evaluate how different characteristics from formalin-fixed paraffin-embedded (FFPE) samples, age of the block or DNA concentration could influence the success of $B R A F$ and NRAS mutational screening.

Methods: DNA was obtained from 144 FFPE samples (62 primary melanoma, 43 sentinel lymph nodes [SLN] and 39

\footnotetext{
*Corresponding author: Dr. Susana Puig, Dermatology Department, Hospital Clinic, C/Villarroel, 170, 08036 Barcelona, Spain, Tel.: 034932275400, Fax: 034932275402,

E-mail: susipuig@gmail.com; Center for Biomedical Network Research on Rare Diseases (CIBERER), Valencia, Spain; and University of Barcelona, Barcelona, Spain

Miriam Potrony: Dermatology Department, Melanoma Unit, Hospital Clinic of Barcelona, IDIBAPS, Barcelona, Spain

Celia Badenas and Joan Anton Puig-Butille: Melanoma Unit, Hospital Clinic of Barcelona, Molecular Biology and Genetics Department, Barcelona, Spain; and Center for Biomedical Network Research on Rare Diseases (CIBERER), Valencia, Spain Bénédicte Naerhuyzen and Nicolas Delahaye: GSK Vaccines, Rixensart, Belgium

Paula Aguilera and Gemma Tell-Marti: Melanoma Unit, Hospital Clinic of Barcelona, Dermatology Department, IDIBAPS, Barcelona, Spain; and Center for Biomedical Network Research on Rare Diseases (CIBERER), Valencia, Spain Alba Díaz and Llucia Alos: Hospital Clinic of Barcelona, Pathology Department, IDIBAPS, Barcelona, Spain; and University of Barcelona, Barcelona, Spain

Cristina Carrera and Josep Malvehy: Melanoma Unit, Hospital Clinic of Barcelona, Dermatology Department, IDIBAPS, Barcelona, Spain; Center for Biomedical Network Research on Rare Diseases (CIBERER), Valencia, Spain; and University of Barcelona, Barcelona, Spain
}

metastasis). BRAF and NRAS were sequenced by Sanger sequencing.

Results: Complete sequencing results were obtained from $75 \%$ (108/144) of the samples, and at least one gene was sequenced in $89 \%$ (128/144) of them. BRAF was mutated in $55 \%(29 / 53)$ and $N R A S$ in $11 \%(5 / 45)$ of the primary melanomas sequenced. DNA concentration correlated with the tumor area used for DNA extraction $\left(\mathrm{mm}^{2}\right)$ (adj $p$-value $<0.01, r=0.73)$. The age of the block did not affect sequencing success. In $60 \%$ of samples kept for more than 10 years, both $B R A F$ and NRAS were successfully sequenced.

Conclusions: Preserving sufficient tumor area in FFPE blocks is important. It is necessary to keep the FFPE blocks, no matter their age, as they are necessary to decide the best treatment for the melanoma patient.

Keywords: BRAF; FFPE; melanoma; NRAS; somatic mutations.

\section{Introduction}

Melanoma is the most deadly of the common skin cancers. Melanoma mortality is around $20 \%$, but when the tumor is diagnosed with a thickness exceeding $4 \mathrm{~mm}$, the mortality rises to $60 \%$ [1]. Targeted therapy has become one of the most important strategies for the treatment of metastatic patients [2].

The MAPK-ERK pathway, which includes the RAF, MEK and ERK protein kinases and the small $G$ protein RAS, plays an important role in melanoma development and progression [3]. Mutations in BRAF are found in approximately $50 \%$ of melanomas [4]. BRAF p.V600E is found in up to $90 \%$ of $B R A F$ mutated tumors [5], which allowed the development of targeted therapies against this mutated 
protein. BRAF-inhibitors now play an important role in targeted therapy in melanoma [6]. NRAS is the second most commonly mutated MAPK-related gene in melanoma tumors (13-22\%) [7]. To date it has been very difficult to target this molecule. Furthermore, NRAS mutations can mediate BRAF-inhibitor resistance [8]. New primary melanomas, BRAF wild type, can be developed during BRAF inhibition [9-12]. Thus, the detection of mutations in $B R A F$ and NRAS genes in melanoma tumors is crucial to decide the best treatment for metastatic patients and in the analysis of new tumors developed during BRAF inhibition therapy.

Formalin fixation and paraffin embedding (FFPE) is the conventional method for preserving melanoma tumors [13]. We hypothesize that several characteristics of the block and the sample could influence the capacity to correctly detect $B R A F$ and NRAS mutations in melanoma tumors. The aim of this project was to assess whether different parameters evaluated on hematoxylin-eosin stained slides and the age of the block, could affect DNA extraction and sequencing success.

\section{Materials and methods}

\section{Patients and samples}

The study initially included 165 FFPE samples belonging to 96 melanoma patients from four groups: stage II free of relapse, stage II with relapse, stage III free of relapse and stage III with relapse, respectively (Figure 1). Melanoma subtype from pathology reports was available in $86 \%$ of the samples and Breslow thickness in $95 \%$ (Table 1). Samples were collected from January 1994 to December 2011. The study was approved by the Ethical Committee of the Hospital Clinic of Barcelona. The patients gave their written, informed consent.

Different parameters were evaluated on Hematoxylin-Eosin stained slides: the percentage of tumor present in the whole section, tumor cells in tumor area, immune cells in tumor area, stroma cells in tumor area, other cells in tumor area and total tumor area used for DNA extraction $\left(\mathrm{mm}^{2}\right)$. According to these parameters, the number of sections needed per DNA extraction to obtain $50 \mathrm{~mm}^{2}$ of tumor area with at least $50 \%$ of tumor cells was estimated.

The age of the block was also recorded. All tissues had been routinely fixed in $4 \%$ buffered neutral formalin and embedded in paraffin. Formalin fixation was overnight except for weekend and holidays

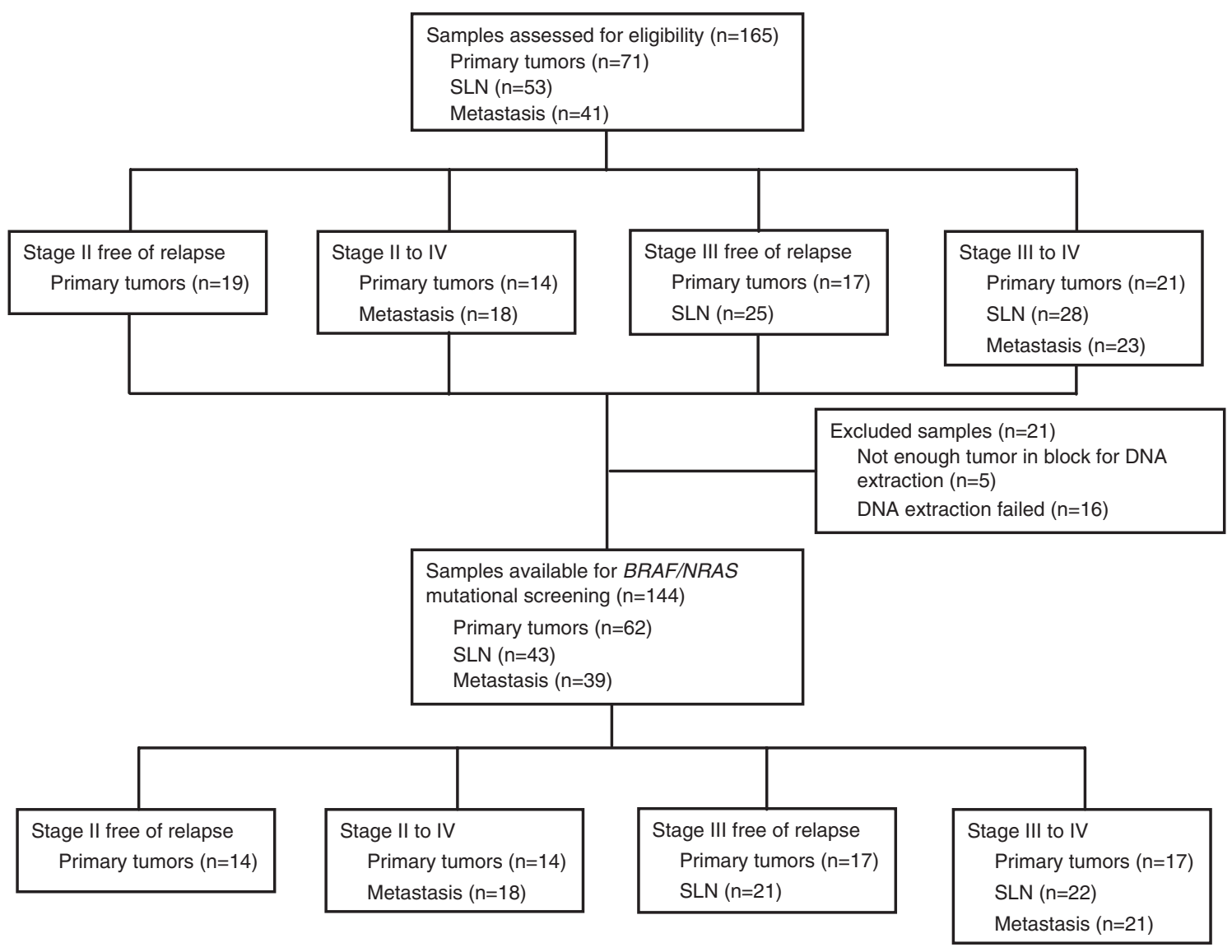

Figure 1: Flowchart of samples included in the study. 
Table 1: Primary tumors description.

\begin{tabular}{|c|c|c|c|c|c|}
\hline \multirow[b]{2}{*}{ Melanoma subtype } & \multicolumn{2}{|l|}{ Stage II } & \multicolumn{2}{|l|}{ Stage III } & \multirow{2}{*}{$\begin{array}{l}\text { Total } \\
\text { n (\%) }\end{array}$} \\
\hline & $\begin{array}{l}\text { Free of relapse } \\
n(\%)\end{array}$ & $\begin{array}{l}\text { With relapse } \\
\text { n (\%) }\end{array}$ & $\begin{array}{l}\text { Free of relapse } \\
n(\%)\end{array}$ & $\begin{array}{l}\text { With relapse } \\
\text { n (\%) }\end{array}$ & \\
\hline SSM & $13(76 \%)$ & $5(63 \%)$ & $15(88 \%)$ & $8(42 \%)$ & $41(67 \%)$ \\
\hline NM & $4(24 \%)$ & $1(12 \%)$ & $2(12 \%)$ & $8(42 \%)$ & $15(25 \%)$ \\
\hline ALM & - & $2(25 \%)$ & - & $1(5 \%)$ & $3(5 \%)$ \\
\hline LMM & - & - & - & $1(5 \%)$ & $1(1.5 \%)$ \\
\hline \multirow[t]{2}{*}{ Unclassifiable } & - & - & - & $1(5 \%)$ & $1(1.5 \%)$ \\
\hline & Mean (SD) & Mean (SD) & Mean (SD) & Mean (SD) & Mean (SD) \\
\hline Breslow (mm) & $3.22(1.59)$ & $5.4(3.89)$ & $1.72(1.12)$ & $4.31(4.54)$ & 3.53 (3.29) \\
\hline
\end{tabular}

Unknown subtype: 2 Stage II free of relapse, 6 Stage II with relapse and 2 Stage III with relapse.

SSM, superficial spreading melanoma; NM, nodular melanoma; ALM, acral lentiginous melanoma; LMM, lentigo malignant melanoma.

where fixation could last 2-3 days. FFPE samples were stored at room temperature.

\section{$B R A F$ and NRAS mutational screening}

DNA extraction was performed using the QIAmp DNA FFPE Tissue Kit (Qiagen, USA). DNA concentration was calculated using the Nanodrop (Thermo Fisher Scientific Inc., USA) absorbance measure method.

$B R A F$ exons 11 and 15, and NRAS exons 2 and 3 were amplified by PCR using the PCR Master Mix (Promega, USA) with the primers and conditions described in the Supplementary Material. For samples that did not amplify in the first PCR, internal primers were designed for each exon to perform nested PCR (Supplementary Material).

The same primers were used for sequencing. The Sanger sequencing process was carried out by Macrogen (Macrogen Europe, The Netherlands) and sequences were analyzed using the SeqPilot Software (JSI Medical Systems GmbH, Germany). If sufficient DNA was available after BRAF and NRAS sequencing, a fluorescent allelespecific PCR followed by capillary electrophoresis was performed to detect the BRAF p.V600E mutation, as previously described [14]. The sample was considered positive for p.V600E only after this variant was confirmed at least twice. The limitation of this study is that the number of repeats needed to obtain the sequence for each sample was not considered.

\section{Statistical analyses}

Different characteristics of the sample were evaluated according to sample type, age of the block ( $<5$ years, 5 to 10 years, $>10$ years), sequencing success defined as having a sequence that could be clearly evaluated (both $B R A F$ and NRAS sequenced - if at least exon 15 of $B R A F$ and exon 3 of NRAS were correctly sequenced it was considered successful - only one gene sequenced, neither gene successfully sequenced) and mutational status of the samples (BRAF/NRAS WT vs. mutated). Sequencing success and mutational status was also evaluated according to the age of the block. To compare means, nonparametric tests (Kruskal-Wallis) were used as continuous variables did not follow a Normal distribution. The Pearson's correlation and the two-sided Fisher's exact test were used for continuous or categorical variables analyses, respectively. All $\mathrm{p}$-values were corrected using the false discovery rate method (http://www.sdmproject. com/utilities/?show=FDR).

\section{Results}

\section{Mutational analyses}

Of the 165 total samples assessed for eligibility, DNA was correctly extracted in $87 \%$ (144/165), DNA extraction failed in 10\% (16/165) and 3\% (5/165) samples had not enough tumor in the block (Figure 1). From samples in which DNA extraction failed, 6/16 were primary tumors, 9/16 SLN and 1/16 a metastasis. The age of block did not affect the DNA extraction success (Table 2). The median area used for DNA extraction was significantly smaller in these samples $(3.8 \mathrm{~mm}$ ) compared to the ones with DNA available (6.67 mm) (adj. $\mathrm{p}=0.01)$.

From the 144 samples with DNA available, $B R A F$ sequencing results were obtained from 87\% (125/144). Overall, 55\% (29/53) of primary tumors sequenced, 49\% (20/41) of SLN and 42\% (13/31) of metastases had mutations in $B R A F$. We identified the p.V600E mutation in $98 \%(61 / 62)$ of mutant BRAF samples and p.V600M in

Table 2: DNA extraction success according the age of block.

\begin{tabular}{lrrr}
\hline Age of block & $\begin{array}{r}\text { Succeeded } \\
\mathbf{n}(\%)\end{array}$ & $\begin{array}{r}\text { Failed } \\
\mathbf{n}(\%)\end{array}$ & $\begin{array}{r}\text { Adjusted } \\
\mathbf{p} \text {-value }\end{array}$ \\
\hline$<5$ years & $37(25.7)$ & $4(25.0)$ & \\
5 to 10 years & $73(50.7)$ & $9(56.3)$ & 1.000 \\
$>10$ years & $34(23.6)$ & $3(18.8)$ & \\
Total & $144(90.0)$ & $16(10.0)$ & - \\
\hline
\end{tabular}


the remaining case. We did not detect mutations in exon 11 of $B R A F$. We sequenced NRAS in $80 \%(115 / 143)$ of all samples. We detected NRAS mutations in $11 \%(5 / 45)$ of the primary tumors, $12 \%$ (5/41) SLN and 14\% (4/29) metastasis. p.Q61R was the most frequent NRAS mutation (35.7\%, 5/14) followed by p.Q61K (28.6\%, 4/14), p.Q13R (21.4\%, $3 / 14$ ) and both p.Q61H and p.G60R were detected in one sample each.

We assessed the concordance of the mutational results between samples from the same patient (Figure 2). There was a concordance between the genetic result of the primary tumor and the SLN or metastasis in 77\% (17/24) and $85 \%(11 / 13)$ of cases, and between SLN and metastasis in 44\% (4/9) of cases, respectively.

We did not detect any statistically significant differences between the different parameters assessed in FFPE samples according to sequencing results or age of the block (Table S1). The area used for DNA extraction was higher in metastasis, which also had a higher amount of tumor cells (adj. $\mathrm{p}<0.01$ ) and a higher DNA concentration (adj. $p=0.02$ ), than primary tumor or SLN. We did not find statistically significant differences between the age of the block and the success of the sequencing process or the capacity to detect mutations in BRAF and NRAS (Table 3). DNA concentration correlated with total tissue area used for DNA extraction (adj. p<0.01, r=0.73), \% of tumor in whole section (adj. $\mathrm{p}<0.01, \mathrm{r}=0.40$ ) and a non-significant tendency was observed in $\%$ of tumor cells in tumor area (adj. $\mathrm{p}=0.06$, $\mathrm{r}=0.19$ ).

\section{Discussion}

Here we have evaluated how different characteristics of FFPE samples, including DNA concentration, influence the success of mutational screening of BRAF and NRAS, key genes for choosing appropriate targeted therapy treatment in advanced melanoma. We selected 165 FFPE samples, including primary melanomas, SLN and metastasis that were paired in some cases. DNA was obtained in $87 \%$ of samples. DNA extraction failure was associated with a smaller tumor area but not with the age of the block. Furthermore, the parameter that best correlated with DNA concentration was the tumor area used for DNA extraction. Thus, when possible, we should preserve large tumor areas in the FFPE blocks.

From 144 samples with DNA, BRAF was sequenced in $87 \%$ of samples and NRAS in $80 \%$. The frequency of genomic mutations detected in primary tumors correlated with that previously reported $[4,15]$. Shitara et al. showed that laser microdissection does not alter the frequency of BRAF and NRAS mutations [15]. We observed discordances between mutations found in the primary melanoma and the SLN and/or the metastasis in some cases. This evidence has already been reported [16]. As previously observed [15, 17-19], in some samples we have detected co-occurrence of $B R A F$ and NRAS mutations. At the single cell level, mutations in $B R A F$ or NRAS are mutually exclusive [17]. Supporting this hypothesis, in the paired SLN of a primary tumor with BRAF p.V600E and NRAS mutation co-occurrence, only a NRAS mutation was detected.
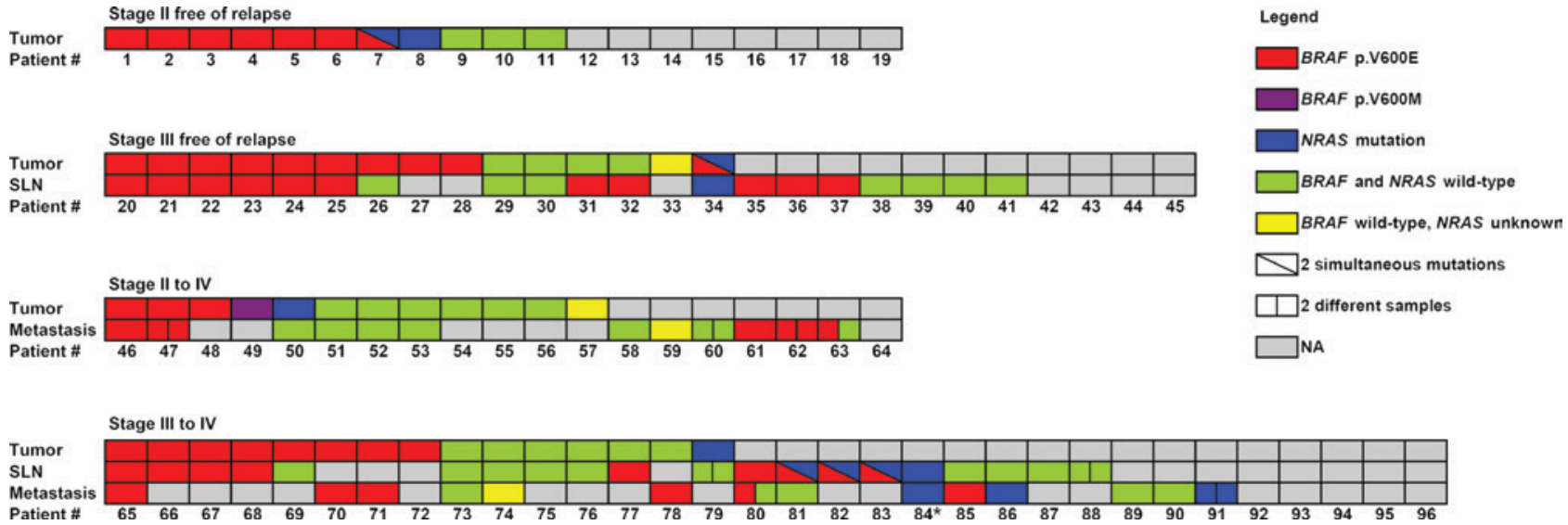

Figure 2: Genetic results by patient and samples.

The Figure shows the genetic results detected by sample type and patient to illustrate the mutation concordance between samples from the same individual. Patients are distributed according to the different staging groups: stage II free of relapse, stage III free of relapse, stage II that progressed to stage IV and stage III that progressed to stage IV. NRAS mutations detected were: p.Q13R, p.G60R, p.Q61R, p.Q61K, p.Q61H. *In Patient \#84 different NRAS mutations were detected in the SLN and the metastasis. Patient \#: Number given to each patient. SLN, sentinel lymph node. NA: indicates samples with no genetic results (includes samples assessed for eligibility but excluded, those in which the sequence could not be properly analyzed and those blocks that were not available for the study). 
Table 3: Sequencing results according to the age of the block.

\begin{tabular}{lrrrr}
\hline & $\begin{array}{r}<5 \text { years } \\
(\mathrm{n}=37) \\
\mathrm{n}(\%)\end{array}$ & $\begin{array}{r}5-10 \text { years } \\
(\mathrm{n}=73) \\
\mathrm{n}(\%)\end{array}$ & $\begin{array}{r}>10 \text { years } \\
(\mathrm{n}=34) \\
\mathrm{n}(\%)\end{array}$ & $\begin{array}{r}\text { Adjusted } \\
\text { p-value }\end{array}$ \\
\hline $\begin{array}{l}\text { Sequencing success } \\
\text { All genes }\end{array}$ & $31(83.8)$ & $59(80.8)$ & $21(61.8)$ & 0.13 \\
One gene & $4(10.8)$ & $5(6.8)$ & $8(23.5)$ & \\
$\quad$ None & $2(5.4)$ & $9(12.3)$ & $5(14.7)$ & \\
$\begin{array}{l}\text { BRAF status } \\
\text { WT }\end{array}$ & $20(57.1)$ & $34(54.0)$ & $9(33.3)$ & 0.27 \\
mutated & $15(42.9)$ & $29(46.0)$ & $18(66.7)$ & \\
NRAS status & & & & \\
WT & $27(84.4)$ & $54(90.0)$ & $20(87.0)$ & 0.76 \\
mutated & $5(15.6)$ & $6(10.0)$ & $3(13.0)$ & \\
\hline
\end{tabular}

The sequencing success is defined as having a sequence that could be clearly evaluated. Adjusted p-values were calculated using the false discovery rate method.

It is recommended to perform the molecular screening on tumor tissue, preferably derived from the metastasis [20]. However, as BRAF inhibitors may induce metastasis in $R A S$ mutant tumors [21], it would be advisable to test both the primary melanoma and the different metastasis tissue available to ensure that there are no different driver mutations within samples that could influence the treatment's success.

Even though more pathology colleges/societies recommend archiving FFPE tumors for at least 10 years [2224] or even 30 years [25], it is not regulated by law in most Countries. There is no single regulation that covers FFPE tissue specimens in the US [26] and in Spain the requirement is of 5 year storage after the last visit of the patient in the institution as part of the clinical record (Spanish Law 41/2002, 14th November). However, a melanoma patient may develop metastasis 10 or more years after the primary tumor diagnosis and having the FFPE specimen preserved could be essential for the selection of the most appropriate treatment. A previous study with FFPE samples from other solid tumors showed that macromolecules could be extracted from FFPE samples, and in terms of quality and quantity did not differ between the samples archived for 1-2 years, 5-7 years or 11-12 years [27]. However, that study did not assess the applicability of those macromolecules obtained for molecular diagnosis. To determine whether the blocks should be kept for a long time for molecular diagnosis in melanoma, we evaluated whether the age of the block affected the success of the sequencing process and the capacity to detect mutations. Using a representative number of blocks with ages $<5$ years old, between 5 and 10 years or over 10 years, we did not detect a diminished capacity to correctly perform the mutational screening of $B R A F$ and NRAS. Although a non-significant decreased percentage of samples with both genes correctly sequenced was observed, we could obtain the sequencing results of NRAS and BRAF in more than $60 \%$ of samples kept for more than 10 years in FFPE blocks.

The optimization of the sequencing process, the existing drug combinations and the development of new drugs will play an important role in the future of melanoma therapy [28]. Some strategies have been developed to cryopreserve melanoma samples in bio-banking with wellestablished mapping protocols using imaging techniques [29], but these types of strategies are not common. Thus, keeping the blocks for more than 10 years is useful for any future genetic analysis that may be required if a patient develops metastasis, to better determine the appropriate treatment.

In conclusion, it is important to preserve FFPE blocks in order to look for mutations that could be targeted by existing or new therapies. Furthermore, the age of the block seems not to influence mutation detection according to the results of this study, even when they have been stored for more than 10 years.

Acknowledgments: Thanks to our patients and their families who are the main reason for our studies; to nurses from the Melanoma Unit of Hospital Clínic of Barcelona, Daniel Gabriel, Pablo Iglesias and Maria E Moliner for helping to collect patient data and to Helena Kruyer for helping with English editing and correction of the manuscript. The work was carried out at the Esther Koplowitz Centre, Barcelona, Spain.

Author contributions: All the authors have accepted responsibility for the entire content of this submitted manuscript and approved submission.

Research funding: GlaxoSmithKline Biologicals SA was the funding source for this study. The research at the Melanoma Unit in Barcelona was/is partially funded by Grants 03/0019, 05/0302, 06/0265, 09/1393 and 12/00840 from Fondo de Investigaciones Sanitarias, Spain; by the CIBER de Enfermedades Raras of the Instituto de Salud Carlos III, Spain; by the AGAUR 2014_SGR_603 of the Catalan Government, Spain; by a grant from "Fundació La Marató de TV3, 201331-30", Catalonia, Spain; by the European Commission under the 6th Framework Program, Contract no: LSHC-CT-2006-018702 (GenoMEL) and by the National Cancer Institute (NCI) of the US National Institute of Health (NIH) (CA83115). Miriam Potrony is the recipient of a PhD Fellowship FI14/00231 (PFIS) from Instituto de Salud Carlos III, Spain. 
Employment or leadership: Bénédicte Naerhuyzen and Nicolas Delahaye are employees of the GSK group of companies. The remaining authors declare no conflicts of interest.

Honorarium: None declared.

Competing interests: The funding organization(s) played no role in the study design; in the collection, analysis, and interpretation of data; in the writing of the report; or in the decision to submit the report for publication.

\section{References}

1. Balch CM, Gershenwald JE, Soong SJ, Thompson JF, Atkins MB, Byrd DR, et al. Final version of 2009 AJCC melanoma staging and classification. J Clin Oncol 2009;2736:6199-206.

2. Eggermont AM, Spatz A, Robert C. Cutaneous melanoma. Lancet 2014;3839919:816-27.

3. Lopez-Bergami P. The role of mitogen- and stress-activated protein kinase pathways in melanoma. Pigment Cell Melanoma Res 2011;245:902-21.

4. Davies H, Bignell GR, Cox C, Stephens P, Edkins S, Clegg S, et al. Mutations of the BRAF gene in human cancer. Nature 2002;4176892:949-54.

5. Flaherty KT, McArthur G. BRAF, a target in melanoma: implications for solid tumor drug development. Cancer 2010;11621:4902-13.

6. Jang S, Atkins MB. Treatment of BRAF-mutant melanoma: the role of vemurafenib and other therapies. Clin Pharmacol Ther 2014;951:24-31.

7. Fernandez-Medarde A, Santos E. Ras in cancer and developmental diseases. Genes Cancer 2011;23:344-58.

8. Kaplan FM, Kugel CH, 3rd, Dadpey N, Shao Y, Abel EV, Aplin AE. SHOC2 and CRAF mediate ERK1/2 reactivation in mutant NRAS-mediated resistance to RAF inhibitor. J Biol Chem 2012;28750:41797-807.

9. Carrera C, Puig-Butille JA, Tell-Marti G, Garcia A, Badenas C, Alos L, et al. Multiple BRAF wild-type melanomas during dabrafenib treatment for metastatic BRAF-mutant melanoma. JAMA Dermatol 2015;1515:544-8.

10. Zimmer L, Hillen U, Livingstone E, Lacouture ME, Busam K, Carvajal RD, et al. Atypical melanocytic proliferations and new primary melanomas in patients with advanced melanoma undergoing selective BRAF inhibition. J Clin Oncol 2012;3019: 2375-83.

11. Dalle S, Poulalhon N, Thomas L. Vemurafenib in melanoma with BRAF V600E mutation. N Engl J Med 2011;36515:1448-9; author reply 50.

12. Debarbieux S, Dalle S, Depaepe L, Poulalhon N, Balme B, Thomas L. Second primary melanomas treated with BRAF blockers: study by reflectance confocal microscopy. $\mathrm{Br} J$ Dermatol 2013;1686:1230-5.

13. Curry JL, Davies MA, Calderone TL, Nathanson K, Prieto VG, Gershenwald JE. Tissue resources for clinical use and marker studies in melanoma. Methods Mol Biol 2014;1102:679-95.

14. Cushman-Vokoun AM, Stover DG, Zhao Z, Koehler EA, Berlin JD, Vnencak-Jones $\mathrm{CL}$. Clinical utility of KRAS and BRAF mutations in a cohort of patients with colorectal neoplasms submitted for microsatellite instability testing. Clin Colorectal Cancer 2013;123:168-78.

15. Shitara D, Tell-Marti G, Badenas C, Enokihara MM, Alos L, Larque AB, et al. Mutational status of nevus associatedmelanomas. Br J Dermatol 2015;173:671-80.

16. Colombino M, Capone M, Lissia A, Cossu A, Rubino C, De Giorgi $\mathrm{V}$, et al. BRAF/NRAS mutation frequencies among primary tumors and metastases in patients with melanoma. J Clin Oncol 2012;3020:2522-9.

17. Sensi M, Nicolini G, Petti C, Bersani I, Lozupone F, Molla A, et al. Mutually exclusive NRASQ61R and BRAFV600E mutations at the single-cell level in the same human melanoma. Oncogene 2006;2524:3357-64.

18. Chiappetta C, Proietti I, Soccodato V, Puggioni C, Zaralli R, Pacini L, et al. BRAF and NRAS mutations are heterogeneous and not mutually exclusive in nodular melanoma. Appl Immunohistochem Mol Morphol 2015;23:172-7.

19. Ekedahl H, Cirenajwis H, Harbst K, Carneiro A, Nielsen K, Olsson $\mathrm{H}$, et al. The clinical significance of BRAF and NRAS mutations in a clinic-based metastatic melanoma cohort. Br J Dermatol 2013;1695:1049-55.

20. Dummer R, Hauschild A, Guggenheim M, Keilholz U, Pentheroudakis G. Cutaneous melanoma: ESMO Clinical Practice Guidelines for Diagnosis, Treatment and Follow-up. Ann Oncol 2012;23(Suppl 7):vii86-91.

21. Sanchez-Laorden B, Viros A, Girotti MR, Pedersen M, Saturno G, Zambon A, et al. BRAF inhibitors induce metastasis in RAS mutant or inhibitor-resistant melanoma cells by reactivating MEK and ERK signaling. Sci Signal 2014;7318:ra30.

22. Sociedad española de Anatomía Patológica - International Academy of Pathology (SeAP-IAP). Libro Blanco de la Anatomía Patológica en España, 4th ed. Spain: Gráficas Dosbi, S. L., 2015.

23. Generalitat de Catalunya - Departament de Sanitat i Seguretat Social. Requisits que han de complir els laboratoris d'anatomia patològica per a la seva autorització. Barcelona: Government online document, 2003.

24. Comité consultatif en anatomopathologie. Guide sur l'assurance qualité en anatomopathologie Phases pré-analytique et analytique. Quebec: Gouvernement du Québec, 2011.

25. The Royal College of Pathologists. The retention and storage of pathological records and specimens, 5th ed. London: The Royal College of Pathologists, 2015.

26. With CM, Evers DL, Mason JT. Regulatory and ethical issues on the utilization of FFPE tissues in research. Methods Mol Biol 2011;724:1-21

27. Kokkat TJ, Patel MS, McGarvey D, LiVolsi VA, Baloch ZW. Archived formalin-fixed paraffin-embedded (FFPE) blocks: a valuable underexploited resource for extraction of DNA, RNA, and protein. Biopreserv Biobank 2013;112:101-6.

28. Olszanski AJ. Current and future roles of targeted therapy and immunotherapy in advanced melanoma. J Manag Care Pharm 2014;204:346-56

29. Malvehy J, Aguilera P, Carrera C, Salerni G, Lovatto L, Scope A, et al. Ex vivo dermoscopy for biobank-oriented sampling of melanoma. JAMA Dermatol 2013;1499:1060-7.

Supplemental Material: The online version of this article (DOI: 10.1515/cclm-2015-1048) offers supplementary material, available to authorized users. 